\title{
On the Hydrogen Atom Beyond the Born-Oppenheimer Approximation
}

\author{
Jean Michel Sellier*a, K.G. Kapanova ${ }^{a}$ \\ ${ }^{a}$ IICT, Bulgarian Academy of Sciences, Acad. G. Bonchev str. 25A, 1113 Sofia, Bulgaria \\ * jeanmichel.sellier@parallel.bas.bg, \\ jeanmichel.sellier@gmail.com
}

\begin{abstract}
Recently a new formulation of quantum mechanics has been suggested which is based on the concept of signed particles, that is, classical objects provided with a position, a momentum and a sign simultaneously. In this paper, we comment on the plausibility of simulating atomic systems beyond the Born-Oppenheimer approximation by means of the signed particle formulation of quantum mechanics. First, in order to show the new perspective offered by this new formalism, we provide an example studying quantum tunnelling through a simple Gaussian barrier in terms of the signed particle formulation. Then, we perform a direct simulation of the hydrogen atom as a full quantum two-body system, showing that the formalism can be a very promising tool for first-principle-only quantum chemistry.
\end{abstract}

Keywords: Quantum mechanics, Quantum chemistry, Hydrogen atom, Born-Oppenheimer approximation, Signed particles formulation, Quantum Tunnelling

\section{Introduction}

The scientific exploration of the hydrogen atom (consisting of one electron and one proton) has had a profound effect in the development of the quantum theory. In 1913, N. Bohr provides the first quantum model in history, theoretically describing the observed quantum energetic jumps experienced by electrons in an atom. The theory was based on the Plank's concept of quanta, and therefore still constrained to the orthodoxy of classical physics. The Schrödinger equation was later applied to a system, providing a way to discover exactly the electronic energies and orbitals of hydrogen.

A key part of the advances in the field is the introduction of one important approximation, based on the assumption that the motion of nucleus and electrons can be separated. Suggested in 1927 by M. Born and J.R. Oppenheimer, the physical basis for approximation is grounded on the fact that the mass of the proton is considerably higher than that of an electron (about 1836 times the electron mass), which makes the nuclei to move much slower, allowing for its kinetic energy to be neglected [1].

Despite its incredible success in both quantum physics and chemistry, it is known that the Born-Oppenheimer approximation disregards some relevant physical processes. For instance, the effects due to vibronic coupling, responsible for describing the influence of electronic vibrations over the nucleus (and vice-versa), are entirely neglected, and as a consequence limit the study of non-adiabatic processes. Moreover, due to the very different time scales involved in electronic and proton transport, the treatment of an atom as a full quantum many-body object (electrons and protons all together) remains a challenging computational problem [2], [3].

Recently, a new formulation of quantum mechanics based on the concept of signed particles has been introduced [4]. The underlying principle of the formulation utilizes ensembles of classical signed field-less particles in the simulation of time-dependent quantum system. The formalism consist of a set of three postulates, which describe the evolution of a quantum system. Specifically, these rules dictate how an ensemble of particles evolves, one particle at a time (which allows for trivial parallelization since in this context particles are completely independent from each other). This approach is completely equivalent to other (more standard) formulations of quantum mechanics such as, for example, the one suggested by E. Schrödinger (in other words, they provide exactly the same predictions). Importantly, the formulation has been extended to time-dependent, quantum many-body problems showing already to be extremely promising and useful, e.g. in the case of indistinguishable Fermions [5], [6], [7]. 
The purpose of this work is to apply for the first time the signed Particle formalism to the hydrogen atom considered as a time-dependent two-body problem, therefore going beyond the Born-Oppenheimer approximation. To prove our point, we stress on the fact that both the electron and proton are considered as quantum objects and the system is treated as a whole described by the Signed particle formalism.

The paper is organized as follows. In the next section, we introduce the formulation and we present the set of postulates which completely defines the new theory. In order to highlight the new perspective offered by this novel theory, we discuss the formalism in the context of the well-known quantum tunnelling effect. Finally, we show preliminary one-dimensional results from a simulation of a hydrogen atom, showing that quantum physics and chemistry beyond the Born-Oppenheimer approximation is at our reach at a level of details never attained before.

\section{The signed particle formulation}

This section introduces the signed particle formulation of quantum mechanics recently suggested in [4]. It consists of a set of three postulates which are reported below. These postulates, or rules, can be seen as physical laws defining the dynamics of (classical) signed particles in the presence of a given potential. In this context, quantum systems are now described by means of ensembles of signed particles. For the sake of simplicity, we limit the discussion to the case of a one-dimensional real space, although the generalization to higher-dimensional space is possible and relatively easy to obtain.

Postulate I. Physical systems can be described by means of (virtual) Newtonian particles, i.e. provided with a position $\mathbf{x}$ and a momentum $\mathbf{p}$ simultaneously, which carry a sign which can be positive or negative.

Postulate II. A signed particle, evolving in a potential $V=V(\mathbf{x})$, behaves as a field-less classical point-particle which, during the time interval $d t$, creates a new pair of signed particles with a probability $\gamma(\mathbf{x}(t)) d t$ where

$$
\gamma(\mathbf{x})=\int_{-\infty}^{+\infty} \mathcal{D} \mathbf{p}^{\prime} V_{W}^{+}\left(\mathbf{x} ; \mathbf{p}^{\prime}\right) \equiv \lim _{\Delta \mathbf{p}^{\prime} \rightarrow 0^{+}} \sum_{\mathbf{M}=-\infty}^{+\infty} V_{W}^{+}\left(\mathbf{x} ; \mathbf{M} \Delta \mathbf{p}^{\prime}\right),
$$

and $V_{W}^{+}(\mathbf{x} ; \mathbf{p})$ is the positive part of the quantity

$$
V_{W}(\mathbf{x} ; \mathbf{p})=\frac{i}{\pi^{d} \hbar^{d+1}} \int_{-\infty}^{+\infty} d \mathbf{x}^{\prime} e^{-\frac{2 i}{\hbar} \mathbf{x}^{\prime} \cdot \mathbf{p}}\left[V\left(\mathbf{x}+\mathbf{x}^{\prime}\right)-V\left(\mathbf{x}-\mathbf{x}^{\prime}\right)\right],
$$

known as the Wigner kernel (in a d-dimensional space) [8]. If, at the moment of creation, the parent particle has sign $s$, position $\mathbf{x}$ and momentum $\mathbf{p}$, the new particles are both located in $\mathbf{x}$, have signs $+s$ and $-s$, and momenta $\mathbf{p}+\mathbf{p}^{\prime}$ and $\mathbf{p}-\mathbf{p}^{\prime}$ respectively, with $\mathbf{p}^{\prime}$ chosen randomly according to the (normalized) probability $\frac{V_{W}^{+}(\mathbf{x} ; \mathbf{p})}{\gamma(\mathbf{x})}$.

Postulate III. Two particles with opposite sign and same phase-space coordinates (x, $\mathbf{p})$ annihilate.

The obtained physical picture by this novel approach is rather peculiar compared to the standard quantum mechanics (albeit there is certain resemblance to the Feynman approach). In point of fact, a quantum system is forthwith described by ensembles of field-less, classical (i.e. provided with a position and a momentum simultaneously) particles. In the formalism those point-like objects carry a sign which can be either positive or negative. Their interaction with the potential $V=V(x)$ is realized through particle creation events only. Accordingly, one obtains the macroscopic properties of a quantum system by averaging a macroscopic variable $A=A(x ; p)$ over the ensemble of particles, taking into account their sign, i.e.

$$
<A>=\frac{1}{N} \sum_{i=1}^{N} s_{i} A\left(x_{i} ; p_{i}\right),
$$

where $s_{i}, x_{i}$ and $p_{i}$ are represented by the sign, the position and the momentum, respectively, of the $i$-th particle (just as in classical statistical mechanics, but now taking the sign of the particles into account). 
The postulates are derived from a physical interpretation of the time-dependent Wigner equation which is rewritten in terms of a Fredholm equation of second kind [4], [9]. It is possible to prove that this set of rules is sufficient to reconstruct the time-dependent evolution of any quantum system [4]. In a similar way, one can extend this set of postulates to the case of many-body systems (starting, this time, from the many-body time-dependent Wigner equation) 1 .

Finally, the reader should note that the integral in (1) is not a Riemann integral. In fact, we call it a momentum integral by analogy with the concept of path integral (the former covering the space of momenta, the latter spanning the space of paths). Results coming from a practical implementation of the approach are shown in Fig. 5 for an abrupt potential barrier.

Classical limit, $\hbar \rightarrow 0$. In terms of the classical limit, there are some particular facets worth discussing. It is indicative to note that the creation of signed particles is not allowed in the classical limit [4]. As such, we differentiate between two possible scenarios - the free space and systems in the presence of an external potential. In the first case, the classical limit is trivial since the function $\gamma(x)$ is zero everywhere and, thereupon, no particle creation event transpires at all. In the second scenario, however, i.e. when the potential $V(x) \neq 0$ for some $x$, by introducing the change of integration variable $x^{\prime}=\frac{p}{2 m} t^{\prime}$ and supposing that the potential $V=V(x)$ can be written in terms of a McLaurin series, we obtain the following expression for the Wigner kernel (2)

$$
\begin{aligned}
V_{W}(x ; p) & =\frac{2 p}{\pi \hbar^{2} m} \sum_{n=0, \text { even }}^{+\infty} \int_{-\infty}^{+\infty} d t^{\prime} \cos \left(\frac{p^{2} t^{\prime}}{\hbar m}\right) \frac{V^{(n)}(x)}{n !}\left(\frac{p t^{\prime}}{2 m}\right)^{n} \\
& =\frac{2 p}{\pi \hbar^{2} m} \int_{-\infty}^{+\infty} d t^{\prime} \cos \left(\frac{p^{2} t^{\prime}}{\hbar m}\right) V(x)+ \\
& +\frac{2 p}{\pi \hbar^{2} m} \int_{-\infty}^{+\infty} d t^{\prime} \cos \left(\frac{p^{2} t^{\prime}}{\hbar m}\right) \frac{1}{2} \frac{\partial^{2} V(x)}{\partial x^{2}}\left(\frac{p t^{\prime}}{2 m}\right)^{2}+ \\
& +O\left(\hbar^{4}\right),
\end{aligned}
$$

keeping in mind that the summation is performed over even numbers, and $V^{(n)}(x)$ represents the $n$-th order derivative of $V(x)$. Moreover, by omitting every term down to the second order with respect to $\hbar$ and integrating, one acquires

$$
\begin{aligned}
V_{W}(x ; p) & \approx\left[\frac{2 V(x)}{\pi p \hbar} \sin \left(\frac{p^{2} t^{\prime}}{\hbar m}\right)\right]_{-\infty}^{+\infty} \\
& =2 \lim _{t^{\prime} \rightarrow+\infty} \frac{2 V(x)}{\pi p \hbar} \sin \left(\frac{p^{2} t^{\prime}}{\hbar m}\right) \\
& \approx \frac{4 V(x)}{\pi p \hbar} \sin \left(\frac{p^{2} T}{\hbar m}\right),
\end{aligned}
$$

for a "long enough time" $T$.

Finally, through the combination of this result with formula (1) we obtain the corresponding function $\gamma(x)$ which, now, reads:

$$
\gamma(x)=\lim _{\Delta p \rightarrow 0^{+}} \sum_{M=-\infty}^{+\infty}\left[\frac{4 V(x)}{\pi \hbar(M \Delta p)} \sin \left(\frac{(M \Delta p)^{2} T}{\hbar m}\right)\right]^{+},
$$

with $[\ldots]^{+}$depicting the positive part of the quantity in the brackets.

For the sake of simplicity, supposing that $V(x)>0$ for $\forall x$, the term in brackets is transformed to positive only when

and, for $\hbar \rightarrow 0$, one has

$$
0 \leq M \leq \frac{\hbar m \pi}{\Delta p^{2} T}
$$

$$
0 \leq M \leq 0
$$

\footnotetext{
${ }^{1}$ The interested reader is encouraged to download and study the source code implementing this algorithm from [10].
} 
hence $M=0$. In other words, $\gamma(x)=0$ for $\forall x$, with creation of particles forbidden. The reader should note that the generalization of this result to the many-body case is obtained in a similar manner and leads to the same conclusion.

Quantum Tunnelling. The concept of signed particle formulation with its three postulates provides a new physical picture, distinctive and peculiar compared to the other available formulations of quantum mechanics. Important example to consider comes from the phenomenon of quantum tunnelling. In this work, we consider a one-dimensional electron impinging on a Gaussian potential barrier centered at the origin $x=0$ :

$$
V(x)=-U e^{-\frac{x^{2}}{2 \sigma^{2}}}
$$

with $U$ being a negative value and representing the peak of the barrier, and $\sigma$ expressing the dispersion of the barrier in the real space. In this simple scenario, one can demonstrate by means of standard analytical manipulations of formula (2), that the Wigner kernel reads:

$$
V_{W}(x ; p)=+4 \sqrt{2 \pi} U \sigma e^{-2\left(\frac{\sigma p}{\hbar}\right)^{2}} \sin \left(\frac{2 x p}{\hbar}\right)
$$

(a representation is reported in Fig. 11). Actualizing the fact that $[f(x)]^{+}=\frac{|f(x)|+f(x)}{2}$, one can show that:

$$
\begin{aligned}
{\left[V_{W}(x ; p)\right]^{+} } & =-4 \sqrt{2 \pi} U \sigma e^{-2 \frac{\sigma^{2} p^{2}}{\hbar^{2}}} \times\left[\sin \left(\frac{2 x p}{\hbar}\right)\right]^{+} \\
& =-4 \sqrt{2 \pi} U \sigma e^{-\frac{2 p^{2} \sigma^{2}}{\hbar^{2}}}\left\{\frac{\left|\sin \left(\frac{2 x p}{\hbar}\right)\right|+\sin \left(\frac{2 x p}{\hbar}\right)}{2}\right\},
\end{aligned}
$$

which, by utilizing (1) and the antisymmetry of the sinusoidal function, finally leads to:

$$
\gamma(x)=-4 \sqrt{2 \pi} U \sigma \times \lim _{\epsilon \rightarrow 0} \sum_{M \in \mathbb{N}}\left\{e^{-2 \frac{\sigma^{2}(M \epsilon)^{2}}{\hbar^{2}}}\left|\sin \left(\frac{2 x M \epsilon}{\hbar}\right)\right|\right\} .
$$

It is important to note that the above series can be shown to be convergent by using the following series of inequalities. Indeed, one gets:

$$
0<e^{-\frac{2 M^{2} \epsilon^{2} \sigma^{2}}{\hbar^{2}}}\left|\sin \left(\frac{2 x M \epsilon}{\hbar}\right)\right|<e^{-\frac{2 M^{2} \epsilon^{2} \sigma^{2}}{\hbar^{2}}}<\frac{1}{\left(2 M^{2} \epsilon^{2} \sigma^{2}\right) !} .
$$

Additionally, to enable a physical interpretation of the function $\gamma=\gamma(x)$, we now explicit a few terms of the series (7). In turn it provides:

$$
\begin{aligned}
\gamma(x)=-4 \sqrt{2 \pi} U \sigma \times \lim _{\epsilon \rightarrow 0} & \left\{e^{-2 \frac{\sigma^{2}\left(\epsilon^{2}\right.}{\hbar^{2}}}\left|\sin \left(\frac{2 x \epsilon}{\hbar}\right)\right|+e^{-2 \frac{\sigma^{2}(2 \epsilon)^{2}}{\hbar^{2}}}\left|\sin \left(\frac{4 x \epsilon}{\hbar}\right)\right|\right. \\
+ & e^{-2 \frac{\sigma^{2}(3 \epsilon)^{2}}{\hbar^{2}}}\left|\sin \left(\frac{6 x \epsilon}{\hbar}\right)\right|+e^{-2 \frac{\sigma^{2}(4 \epsilon)^{2}}{\hbar^{2}}}\left|\sin \left(\frac{8 x \epsilon}{\hbar}\right)\right| \\
+ & \ldots\} .
\end{aligned}
$$

It becomes immediately apparent that the exponentials in the formula above tend to damp the various terms with an increasing value $M$. One should note that the damping is not rapid. In fact, for very small $\epsilon$ the two quantities $\sigma M \epsilon$ and $\hbar$ tend to be comparable. As a consequence, the decrease of the series is actualized at a very slow rate, with many terms needing to be taken in the series in order to have a good approximation of the function $\gamma=\gamma(x)$. On the other side, the terms $\left|\sin \left(\frac{2 x M \epsilon}{\hbar}\right)\right|$ contribute with values in the range $[0,1]$ where one can see how these oscillating contributions sum up in Fig. 2. The numerically computed function $\gamma(x)$ is depicted by a (red) continuous curve, which is compared 
to various sums of terms up to 4, 8, 16 and 32 respectively, represented by the (blue) dashed curves. It is clear that despite the slow convergence, the series eventually get closer and closer to the numerical $\gamma(x)$ function. One important observation in regards to the quite unexpected spikes close to the boundaries of the observable domain are actually approached by the converging series of terms. Although there exist a possibility for their incorrect interpretation as numerical spurious oscillations.

Additionally, it is feasible to extract several mathematical properties for the function $\gamma(x)$ which would hardly be visible in a purely numerical context. Indeed, one can easily show that $\gamma(x)=\gamma(-x)$ for all $x \in]-\infty,+\infty[$ and that $\gamma(0)=0$. We should point out that these properties have an interesting physical interpretation. The first one defines the system as symmetric with respect to the creation of signed particles, and something rather presupposed from a symmetric potential (the Gaussian barrier is symmetric with respect to $x=0$ ). The more interesting case comes with the property $\gamma(0)=0$, which elucidates a physical fact otherwise impossible to distinguish through purely numerical arguments. One observes in this case that particle creation is forbidden at the top of the Gaussian barrier and signed particles going through that (very restricted but apparently non-negligible) area behave as field-less classical objects. As such, the area around the top behaves just like a (short) domain with a constant applied potential.

As discussed in the examples above, the Signed particle formulation provides a possible level of detail never attained before. We are now ready to apply the formulation to the simulation of the hydrogen atom as a full quantum system, as discussed in the next section.

\section{Beyond the Born-Oppenheimer approximation}

Considering the hydrogen atom as a (inseparable) two-body system consisting of a (quantum) proton and a (quantum) electron, its time-dependent dynamics is governed by the two-body Wigner equation (in one-dimensional space and Bloch formalism):

$$
\frac{\partial f_{W}}{\partial t}+\frac{\hbar k_{e^{-}}}{m_{e^{-}}} \frac{\partial f_{W}}{\partial x_{e^{-}}}+\frac{\hbar k_{p^{+}}}{m_{p^{+}}} \frac{\partial f_{W}}{\partial x_{p^{+}}}=\hbar^{2} \int d \mathbf{k}^{\prime} f_{W}\left(\mathbf{x} ; \mathbf{k}^{\prime}\right) V_{W}\left(\mathbf{x} ; \mathbf{k}+\mathbf{k}^{\prime}\right)
$$

a partial integro-differential equation where $x_{e^{-}}$and $x_{p^{+}}$are the position of the electron and proton respectively, with $\mathbf{x}=\left(x_{e^{-}}, x_{p^{+}}\right), k_{e^{-}}$and $k_{p^{+}}$representing the wave-number of the electron and proton respectively, with $\mathbf{k}=\left(k_{e^{-}}, k_{p^{+}}\right)$, $f_{W}=f_{W}(\mathbf{x} ; \mathbf{k})$ the Wigner quasi-distribution function of the system, and $V_{W}=V_{W}(\mathbf{x} ; \mathbf{k})$ the Wigner kernel expressing the forces involved along with their non-local nature [8]. If we consider the results from the previous section, we can define the initial conditions for both particles to consist of Gaussian wave-packets where the proton is highly localized in space (and therefore strongly delocalized in wave-space), see Fig. 3 (right-hand side), and the electron is delocalized in both space and wave-space (around $k=0 / \mathrm{nm}$ ), see Fig. 4 (top, right-hand side).

The reader can see the results of the simulation, presented in Figs. 4 and 3 . The left column of Fig. 4 shows the time-dependent evolution of the normalized probability density for the electron (with blue-continuous curve), and for the proton (with red- dashed curve). The times reported (from top to bottom) correspond to 0, 3 and 6 attoseconds respectively. Interestingly, one can notice the emergence of a radius for the electron probability density comparable to the Bohr radius $(\approx 0.529$ Angstrom) after a few attoseconds. The evolution in time of the very same electron in the phase-space (in other words, the electron quasi-distribution function) is shown in the right column of Fig. 4

The observed negative peaks appearing near the point

$(x, k)=(0.20991 \mathrm{~nm},-22.263 / \mathrm{nm})$, represent a definite indication of the Heisenberg principle. While the electron dynamics is very fast (on a time scale of the order of attoseconds), Fig. 3 indicates how the evolution of the proton proceeds comparatively very slowly. Distinctly, the left-hand side plot demonstrates the relative difference between the proton probability densities $\rho_{0}(x)$ and $\rho(x ; t)$ at times $t=0$ as and $t=6$ as, respectively. Here the difference is defined as:

$$
\varepsilon\left(x_{p^{+}}\right)=\frac{\rho\left(x_{p^{+}} ; t=6\right)-\rho_{0}\left(x_{p^{+}}\right)}{\max _{x_{p^{+}} \in D} \rho_{0}\left(x_{p^{+}}\right)},
$$

with $D$ being the spatial domain of the simulation. While the plot shows how slow the proton dynamics is, it, in fact, also appears that the proton wave-packet is evolving in time. This is conceivable due to the vibrational interactions with the electron, an effect that apparently seems to happen even in the hydrogen atom although it remains almost imperceptible. 


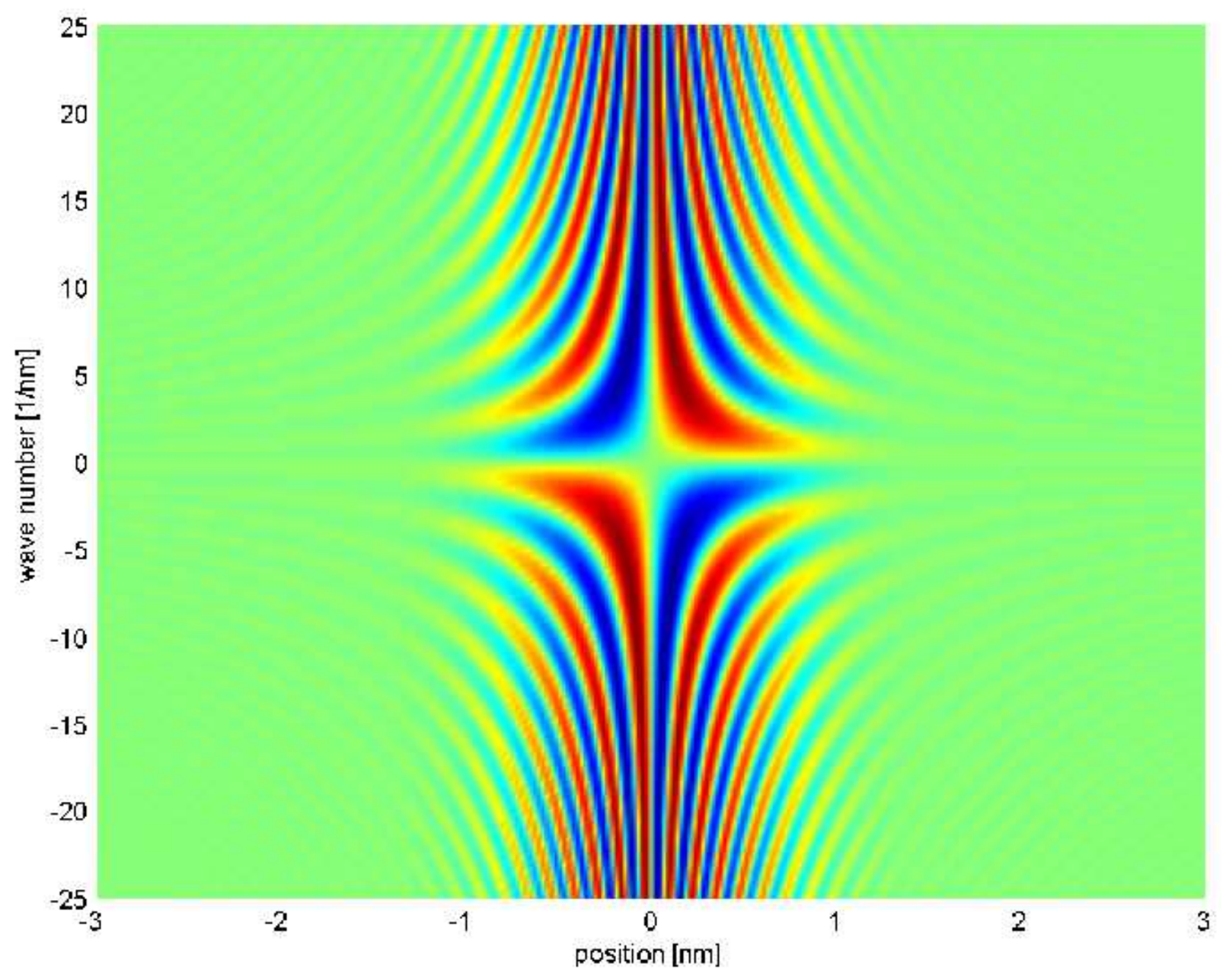

Figure 1: The Wigner kernel $V_{W}(x ; p)$ corresponding to a Gaussian potential barrier. 

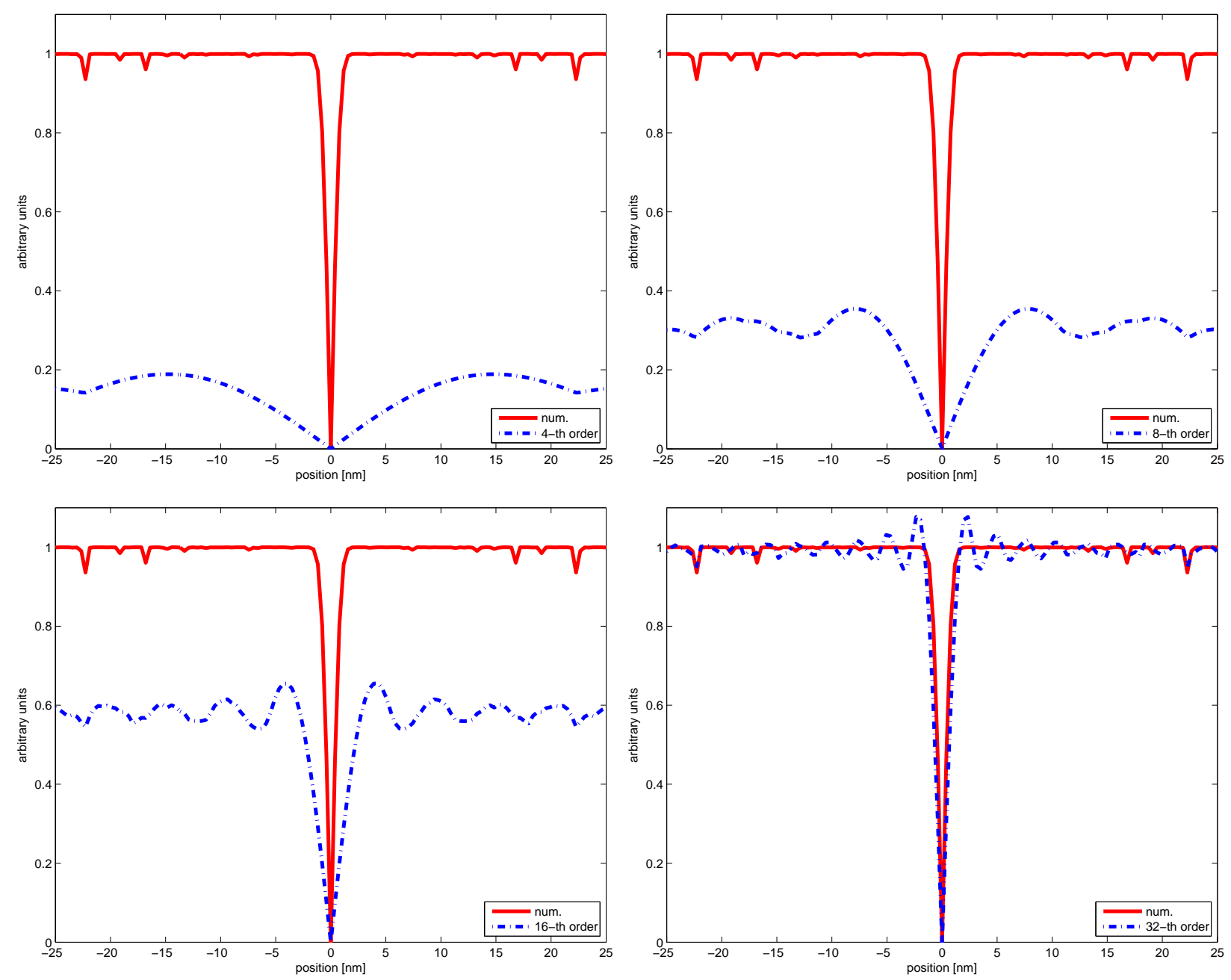

Figure 2: The function $\gamma(x)$ in the case of a Gaussian potential barrier. 

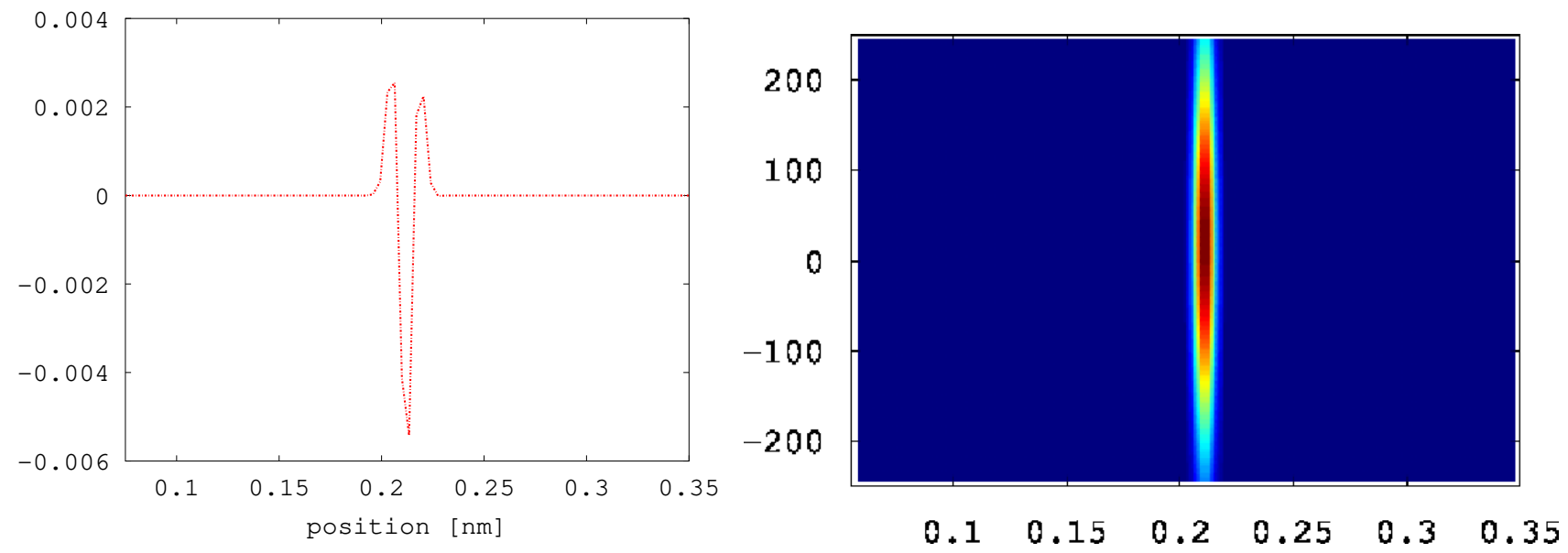

Figure 3: Left: Relative difference corresponding to the proton probability densities $\rho_{0}(x)$ and $\rho(x ; t)$ at times, respectively, $t=0$ as and $t=6$ as. The difference is defined as $\varepsilon(x)=\frac{\rho(x ; t=6)-\rho_{0}(x)}{\max _{x \in D} \rho_{0}(x)}$, with $D$ the spatial domain of the simulation. Right: initial distribution function for the proton. The positions (x-axis) are expressed in nanometer while the wave-numbers (y-axis) are in nanometer ${ }^{-1}$. 

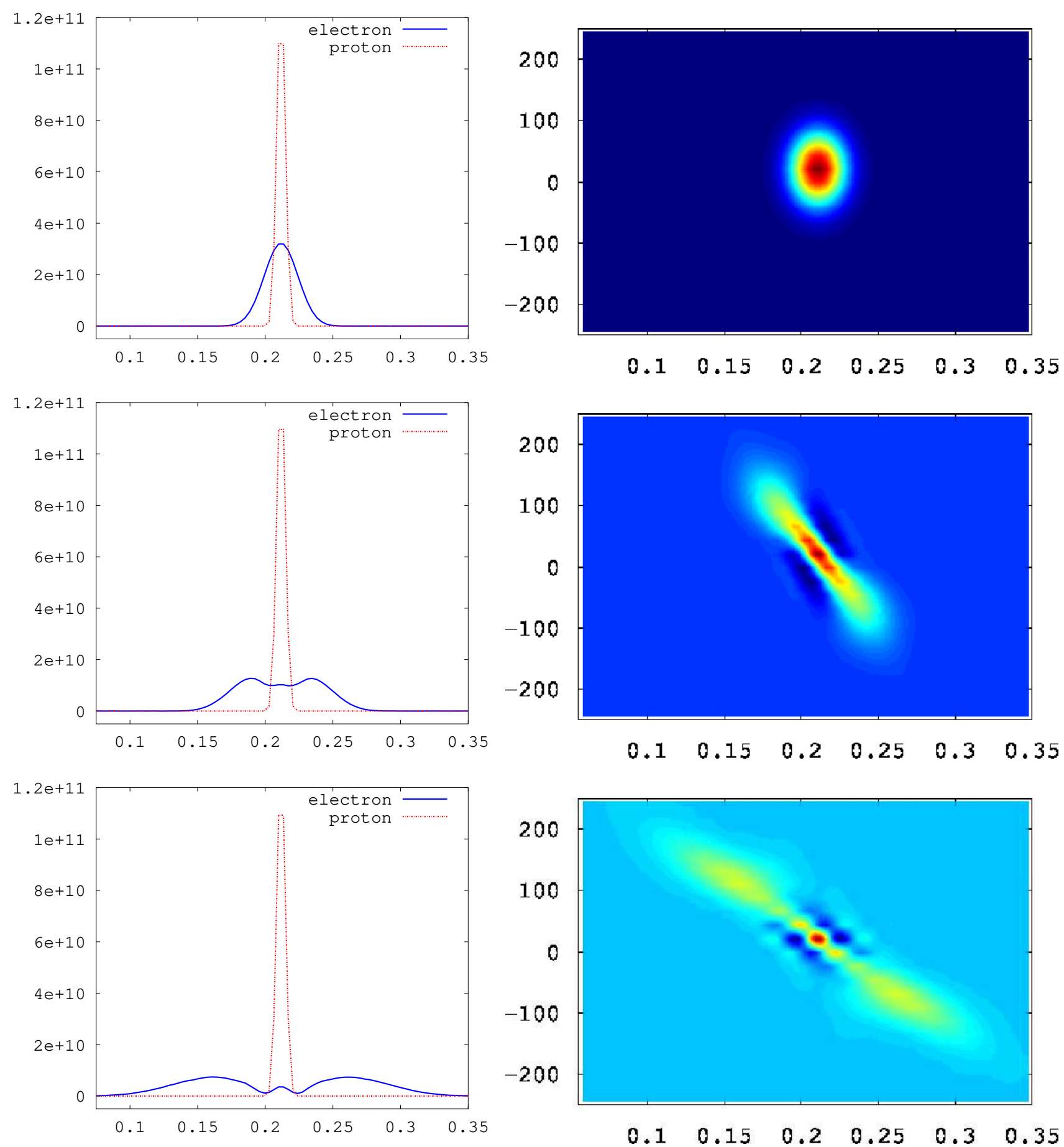

Figure 4: Left column: time-dependent evolution of the (normalized) probability density for the electron, (blue) continuous curve, and proton, (red) dashed curve. The curves (from top to bottom) correspond to the times 0 as, 3 as and 6 as respectively. The appearance of a radius comparable to the Bohr radius is observable after a few attoseconds in the probability density of the electron. Positions ( $\mathrm{x}$-axis) are in nanometers. Right column: evolution in time of the electron quasi-distribution function. Negative peaks appear after several attoseconds, a clear signature of the presence of the Heisenberg principle. Positions (x-axis) are in nanometers, Wave-numbers (y-axis) are in 1/nanometer. 

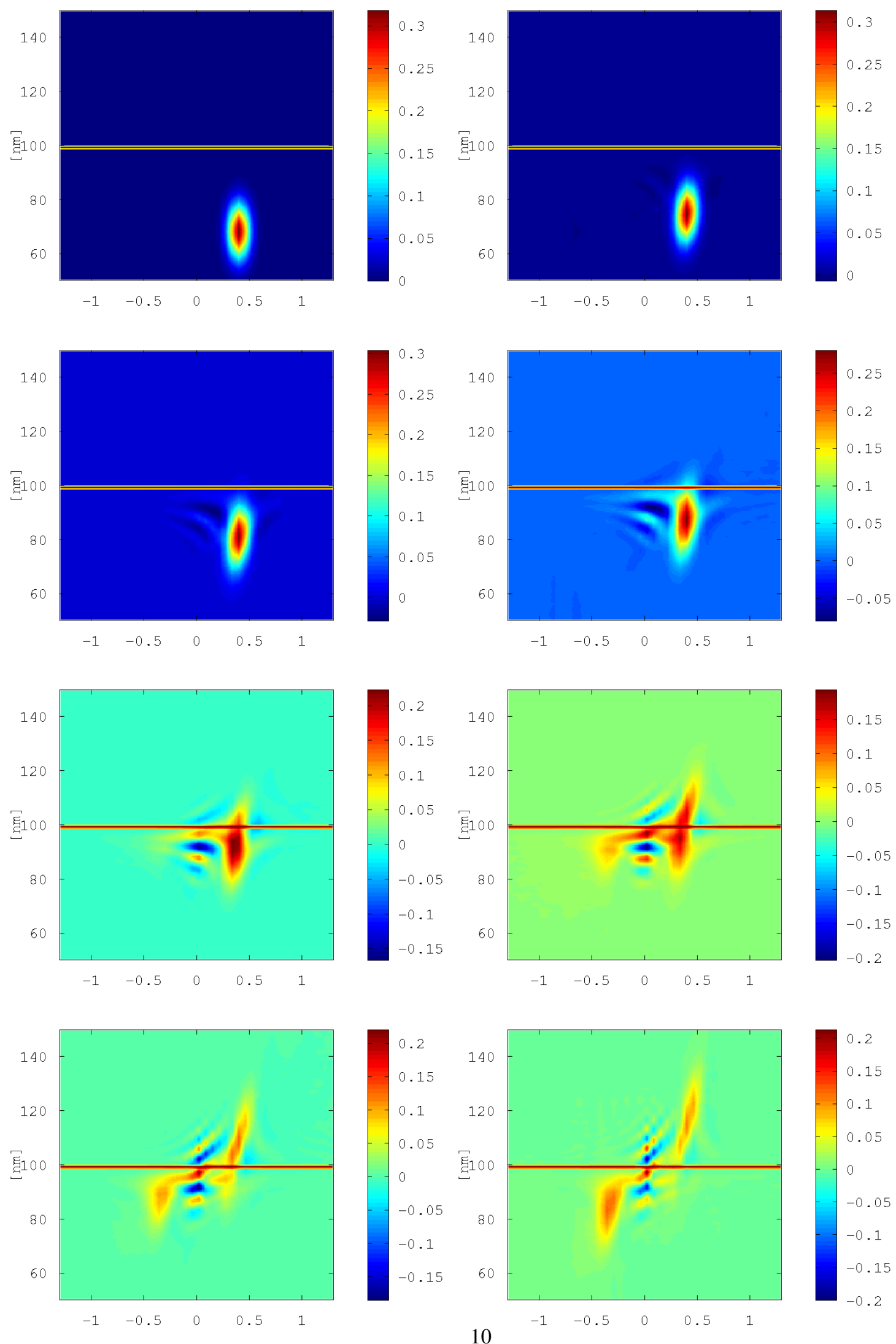

Figure 5: Quantum tunnelling experiment in phase-space (from [4]): an initially Gaussian wave-packet is directed towards a potential barrier equal to $0.10 \mathrm{eV}$. The wave packet is represented by a set of signed particles (initially all positive) which is evolved in time. The times reported are (from left to right, from top to bottom) $0 \mathrm{fs}, 10 \mathrm{fs}, 20 \mathrm{fs}, 30 \mathrm{fs}, 40 \mathrm{fs}, 50 \mathrm{fs}, 60 \mathrm{fs}$ and $70 \mathrm{fs}$ respectively. Negative values (dark blue) appear during the evolution. The position of the potential barrier is schematically represented by the (red) line in the middle of the domain. 


\section{Conclusions}

In this paper, we discussed the application of the recently suggested formulation of quantum mechanics involving the concept of signed particles to the time-dependent simulation of an hydrogen atom beyond the Born-Oppenheimer approximation. We have provided an example studying quantum tunnelling trough a simple Gaussian barrier in terms of the signed particle formulation. We have furthermore shown through the utilization of a set of three simple postulates, how the hydrogen atom can be considered as a whole quantum two-body problem. While this direct simulation is limited to the one-dimensional case, we can certainly draw interesting conclusions about the results of the simulation. We expect that the experiments shown in this work should bring insights into the field of non-adiabatic quantum simulations which are hardly reachable with other methods, and should open a totally new perspective in the field of quantum chemistry. We hope this will encourage other scientists to utilize this formalism for their own scientific investigations.

Acknowledgements. J.M. Sellier acknowledges P. Dollfus, D. Querlioz and S. Shao for the inspiring discussions, and M. Anti for her support and encouragement. K.G. Kapanova has been partially supported by the "Research program for young scientists at the Bulgarian Academy of Science 2017", funded by the Bulgarian Ministry of Education and Science.

\section{References}

[1] M. Born, R. Oppenheimer, Zur quantentheorie der molekeln, Annalen der Physik 389 (20) (1927) 457-484.

[2] D. Marx, J. Hutter, Ab initio molecular dynamics: basic theory and advanced methods, Cambridge University Press, 2009.

[3] D. R. Yarkony, Nonadiabatic quantum chemistry past, present, and future, Chemical reviews 112 (1) (2011) 481-498.

[4] J. M. Sellier, A signed particle formulation of non-relativistic quantum mechanics, Journal of Computational Physics 297 (2015) $254-265$.

[5] J. Sellier, I. Dimov, A wigner monte carlo approach to density functional theory, Journal of Computational Physics 270 (2014) $265-277$.

[6] J. Sellier, I. Dimov, The many-body wigner monte carlo method for time-dependent ab-initio quantum simulations, Journal of Computational Physics 273 (2014) 589-597.

[7] J. Sellier, I. Dimov, On the simulation of indistinguishable fermions in the many-body wigner formalism, Journal of Computational Physics 280 (2015) 287-294.

[8] E. Wigner, On the quantum correction for thermodynamic equilibrium, Physical review 40 (5) (1932) 749.

[9] J. Sellier, M. Nedjalkov, I. Dimov, An introduction to applied quantum mechanics in the wigner monte carlo formalism, Physics Reports 577 (2015) 1-34.

[10] J. Sellier, nano-archimedes, accessed 15 april, 2017, URL: www. nano-archimedes. com. 